\title{
miRNA profiling reveals a potential role of milk stasis in breast carcinogenesis
}

\author{
YI-QI GU ${ }^{1}$, GU GONG ${ }^{2}$, ZHE-LI XU ${ }^{1}$, LI-YING WANG ${ }^{3}$, MING-LI FANG $^{3}$, \\ HUI ZHOU ${ }^{1}$, HUA XING ${ }^{1}$, KE-REN WANG ${ }^{1}$ and LIANG SUN ${ }^{1}$ \\ ${ }^{1}$ Breast Surgery, ${ }^{2}$ Spine Surgery, China-Japan Union Hospital of Jilin University, Changchun, Jilin 130033; \\ ${ }^{3}$ Department of Molecular Biology, College of Basic Medical Sciences, Jilin University, Changchun, Jilin 130021, P.R. China
}

Received December 2, 2013; Accepted February 17, 2014

DOI: $10.3892 /$ ijmm.2014.1677

\begin{abstract}
The tumor microenvironment plays an important role in breast carcinogenesis. Milk acts as an important microenvironment of breast cancer, but its role in breast carcinogenesis is largely unknown. Milk stasis may exist in the breast for a number of years after breastfeeding. In the present study, we reported the first microRNA (miRNA) profiling of milk from patients with milk stasis. We identified 266 known miRNAs and 271 novel miRNAs in 10 milk stasis only samples, 271 known miRNAs and 140 novel miRNAs in 10 milk stasis plus breast neoplasm samples by deep sequencing. miRNA profiles were different between the two groups. Furthermore, nine tumor suppressor miRNAs such as miR-29a, miR-146 and miR-223 were significantly downregulated, while seven oncogenic miRNAs such as miR-451, miR-486, miR-107, miR-92 and miR-10 were significantly upregulated in the milk of milk stasis plus neoplasm patients. Three of the identified miRNAs (miR-140, miR-21 and let-7a) were selected using real-time PCR, confirming that these miRNAs were highly expressed. The results also showed that the three miRNAs detected were more abundant in the milk than in the blood. In summary, the data suggested that miRNAs in milk from milk stasis patients may contribute to breast carcinogenesis and that they are more sensitive biomarkers for breast cancer than miRNAs in the blood.
\end{abstract}

\section{Introduction}

Mammary gland is a highly efficient organ that produces milk during nursing and is composed of epithelial, adipose and other stromal cells (1). Breast cancer is a type of cancer originating

Correspondence to: $\mathrm{Dr}$ Zhe-Li Xu, Breast Surgery, ChinaJapan Union Hospital of Jilin University, No. 126 Xian Tai Road, Changchun, Jilin 130033, P.R. China

E-mail: gyqmary@163.com

Key words: milk stasis, miRNA, miRNA profile, breast cancer, biomarker from breast tissue such as the inner lining of milk ducts or the lobules that supply the ducts with milk (2-4). Breast cancer is 100 times more common in women than in men and accounts for $22.9 \%$ of all cancer types in women $(5,6)$. Therefore, the primary risk factors for breast cancer are female gender and older age $(7,8)$. Other potential risk factors include lack of childbearing or breastfeeding, high hormone levels, diet and obesity $(9,10)$. However, the exact risk factors that contribute to breast cancer development are largely unknown. Although mutations in BRCA genes have been linked to breast cancer initiation, they only account for a small percentage of breast cancers $(11,12)$. These data suggest that the breast microenvironment such as milk may also contribute to breast cancer initiation and development.

MicroRNAs (miRNAs or miRs) are small non-coding single-stranded RNAs transcribed from genomic DNA and processed to mature miRNAs by Drosha in the nucleus and subsequently by Dicer in the cytoplasm $(13,14)$. A variety of miRNAs have been identified to play a role in breast cancer carcinogenesis $(15,16)$. Let-7 family and miR-200 family are relatively well-characterized miRNAs that are crucial to breast cancer initiation and breast cancer cell self-renewal (17-20). In addition, results of a recent study showed that the combination of miR-145 and miR-451 in the blood may discriminate breast cancer patients from healthy controls (21). A notable and important biological feature of miRNAs is their greater stability relative to mRNAs, which makes them stable and powerful biomarkers for breast cancer. Milk and the contained miRNAs act as an important breast cancer microenvironment, however, their roles in breast cancer development are largely undetermined.

It has previously been demonstrated that miRNAs are present in bovine and human breast milk (22-26). miRNAs in breast milk are stable even under extremely acidic conditions ( $\mathrm{pH} \mathrm{1)}$ and the freeze-thawing process, and are resistant to RNase treatment $(22,27)$. Milk is produced but instead of discharging during feeding it remains in the breast, resulting in 'milk stasis' (28-30). The duration required for milk stasis ranges from several years to $\geq 30$ years. Milk stasis in patients is usually found via fiberoptic ductoscopy or operation, with most patients presenting non-spontaneous nipple discharge. Milk stasis is the main cause of mastitis, however, its role in breast cancer remains to be determined. 
The aim of this study was to determine miRNA profiling in milk stasis. Comparison of milk miRNA profiles between patients with milk stasis only and patients with both milk stasis and breast tumor showed differential miRNA expression in the two cohorts, confirmed by quantitative PCR. Our results demonstrated a potential role of milk stasis and the associated miRNAs in breast cancer development.

\section{Materials and methods}

Subject cohorts. Two cohorts of women with milk stasis were enrolled in this study. One cohort included 10 patients with milk stasis but without tumor as evidenced by breast ultrasound and mammography. The other cohort included 10 patients with milk stasis and breast tumor. Pathological examination confirmed that one case was ductal carcinoma in situ, one case was atypical ductal epithelial hyperplasia and eight cases were intraductal papilloma (Fig. 1). All the women involved in this study provided signed informed consent. The study was approved by the Ethics Committees of Jilin University (Changchun, China).

miRNA isolation. Samples were collected by lavaging the breast milk ducts with $0.9 \%$ saline, and frozen until centrifugation in RNAse-free tubes at $4^{\circ} \mathrm{C}$. The substratum liquid layer containing miRNAs was transferred to a new RNAse-free tube, and stored at $-80^{\circ} \mathrm{C}$ until RNA isolation. miRNA isolation was performed using a mirVana miRNA Isolation kit (Ambion, Inc., Foster City, CA, USA). The purity and quantity of isolated miRNA were determined by a spectrophotometer (UV2800 ultraviolet spectrophotometer; Unico, New York, NY, USA).

miRNA identification. Total RNA isolated from the lavage milk obtained from each woman was used for library construction and subjected to single-end sequencing in 36-bp reads using an Illumina Genome Analyzer II (Illumina, San Diego, CA, USA). The raw reads that passed through a series of filters (such as the length and sequence comparison) were termed 'map-pable reads'. The map-pable reads were counted and the identical reads were combined into a single type, and then mapped to the 1,527 human pre-miRNAs registered in the miRBase 18.0 with no more than one mismatch. The most abundant mature variant of a given miRNA was chosen as a reference sequence, which provided the most robust approach for the evaluation of the expression level of miRNA.

Quantitative PCR. cDNA was generated from $5 \mu 1$ total RNA using.One Step PrimeScript ${ }^{\circledR}$ miRNA cDNA Synthesis kit (Takara Bio, Inc., Shiga, Japan) according to the manufacturer's instructions. Quantitative PCR was performed using a High-Specificity miRNA qRT-PCR Detection kit (Takara) on the ABI PRISM ${ }^{\circledR} 7300$ real-time PCR system. The $\Delta \Delta C t$ method was used to determine the expression level of miRNAs in the surveyed samples.

\section{Results}

miRNAs profiling of milk from the two groups. To profile miRNAs in human breast milk from patients with milk stasis (all patients stopped breastfeeding for $\geq 3$ years; average stop
Table I. Summary of known and novel miRNAs in the two groups.

miRNA Novel

Variables miRNA miRNA-5p miRNA-3p precursors miRNA

\begin{tabular}{lccccc}
$\begin{array}{l}\text { Known miRNA } \\
\text { in miRbase 18 }\end{array}$ & 845 & 533 & 542 & 1527 & \\
Milk stasis & 266 & 292 & 292 & 736 & 271 \\
$\begin{array}{l}\text { Milk stasis } \\
\text { plus breast tumor }\end{array}$ & 271 & 292 & 294 & 732 & 140 \\
\hline
\end{tabular}

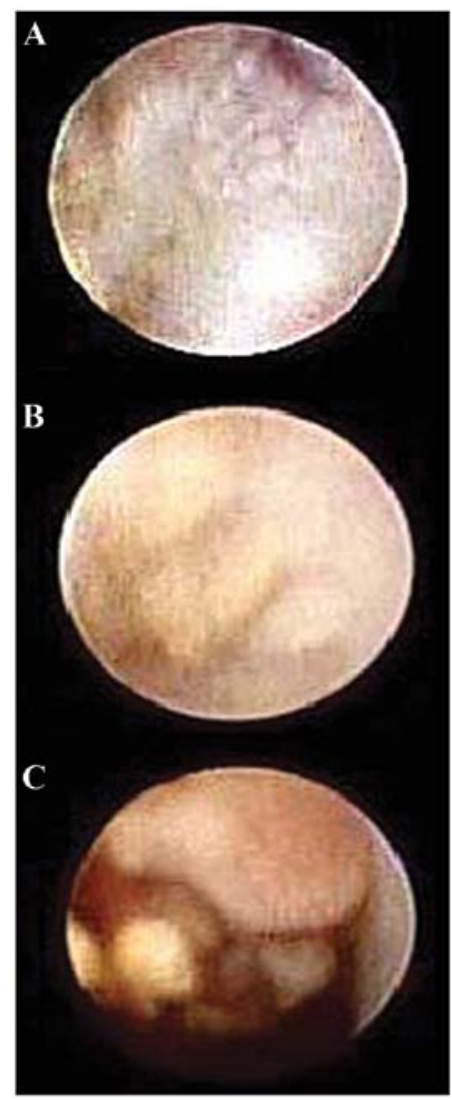

Figure 1. Typical image of different types of neoplasm. (A) Ductal carcinoma in situ, (B) atypical ductal epithelial hyperplasia, and (C) intraductal papilloma.

breastfeeding years, 10; average age, 39.2 years), we extracted total RNA from human saline lavage from breast ducts with milk stasis. The concentration of miRNAs was 88 and $128.4 \mu \mathrm{g} / \mathrm{ml}$ in milk from the milk stasis only group and milk stasis plus breast neoplasm group, respectively.

A total of 821,967 unique sRNAs were detected in 10 milk stasis only samples and a total of 677,177 sRNAs were detected in 10 milk stasis plus breast neoplasma samples. The two groups showed similar length distribution of sRNAs. The majority of sRNAs were at a length of 20, 21, 22 and 23 nt (Fig. 2). A total of $7.56 \%$ unique sRNAs were identified in the two groups, and 41.03 and $51.41 \%$ were specifically detected in the milk stasis 

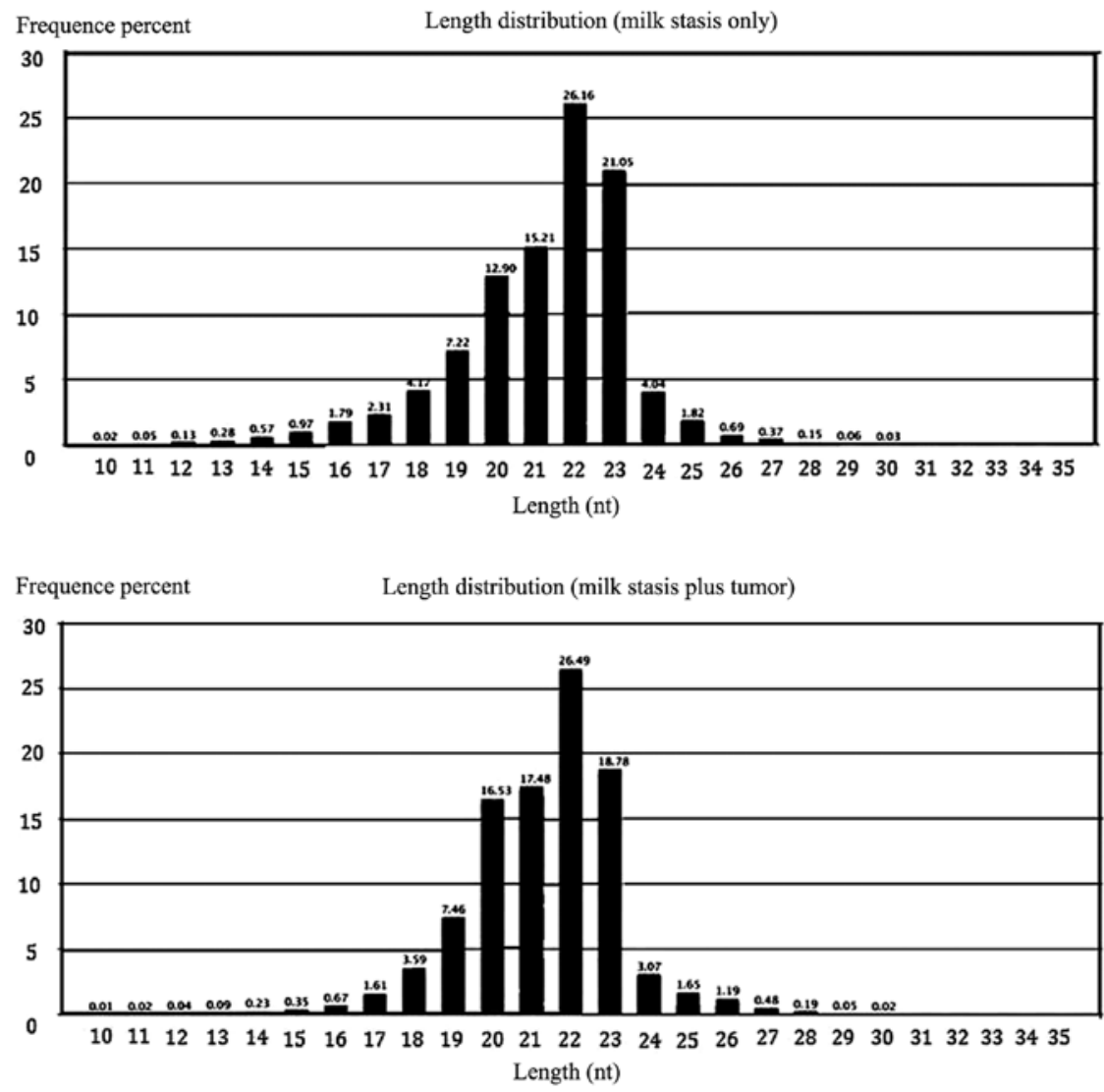

Figure 2. Feature of identified sRNA: length distribution.

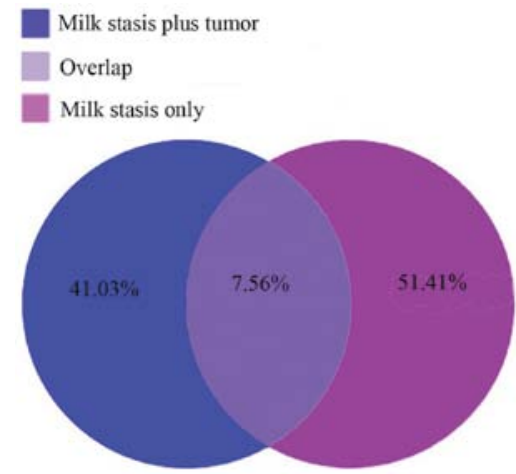

Figure 3. Common and specific sequence between the two groups.

plus breast neoplasm group and milk stasis only group, respectively (Fig. 3). Among them, 266 known miRNAs together with 271 novel miRNAs were detected in 10 milk stasis only samples and 271 known miRNAs together with 140 novel miRNAs were detected in 10 milk stasis plus breast neoplasm samples (Table I). The first nucleotide of miRNAs at a length of 18-30 nt is shown in Fig. 4. Nucleotides A and $U$ were the most frequent first nucleotides.

To understand the features of sRNAs in milk stasis, subtypes of RNAs were identified in the two groups (Fig. 5) and the sequences were aligned to the human genome. A total of $69.82 \%$ of unique sRNA in milk stasis only patients and $67.70 \%$ of unique sRNA in milk stasis plus neoplasm patients were mapped to the genome. Collectively, these data clearly demonstrated that sRNAs are abundant in milk stasis patients. The microenvironment of the epithelial cell was altered during the years following breastfeeding.

Differential expression of miRNA in the two groups. Milk is an important microenviroment for breast cancer development, thus we hypothesized that miRNAs involved in milk stasis may regulate breast cancer development. We compared the expression of miRNAs between milk stasis only patients and milk stasis plus breast neoplasm patients. Fig. 6 shows the differential expression of miRNA in the two groups by plotting of Log2-ratio and scatter plot. A total of 174 known miRNAs showed a differential expression $(\mathrm{p}<0.01)$. Among them, nine miRNAs (hsa-let-7g-5p, hsa-miR-29a-3p, hsa-let-7e-5p, hsa-miR-146a-5p, hsa-miR-146b-5p, hsa-miR-181a-5p, hsa-miR-191-5p, hsa-miR-223-3p, hsa-let-7a-5p) were significantly downregulated $(\mathrm{p}<0.01$, fold-change $>1$ ) compared to milk stasis only patients (Fig. 7A), six of which were tumor suppressors (hsa-let-7g-5p, hsa-miR-29a-3p, hsa-let-7e-5p, hsa-miR-146b-5p, hsa-miR-223-3p, hsa-let-7a-5p). By contrast, seven miRNAs (hsa-miR-451a, hsa-miR-486-5p, hsa-miR107, hsa-miR-16-5p, hsa-miR-185-5p, hsa-miR-92a-3p, hsa-miR-10a-5p) were significantly upregulated $(\mathrm{p}<0.01$, fold-change $>1$ ) in milk stasis plus breast neoplasm patients, compared to milk stasis only patients (Fig. 7B), five of which have an oncogenic function (hsa-miR-451a, hsa-miR-486-5p, hsa-miR-107, hsa-miR-92a-3p, hsa-miR-10a-5p).

We also detected 13 highly expressed miRNAs in the two groups ( $p>0.05$, fold-change $<1)$, nine of which were tumor 

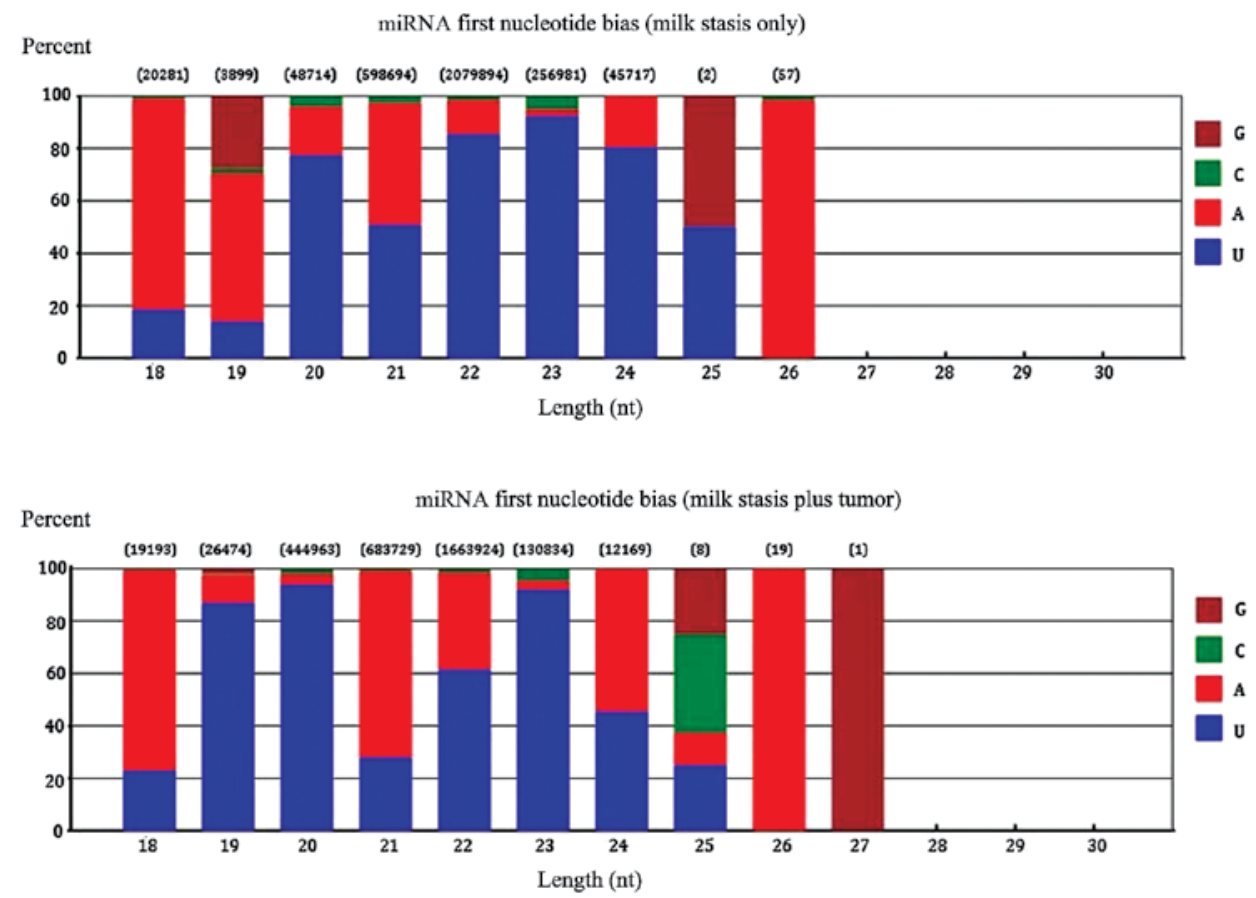

Figure 4. MicroRNA (miRNA) first nucleotide bias in two groups.

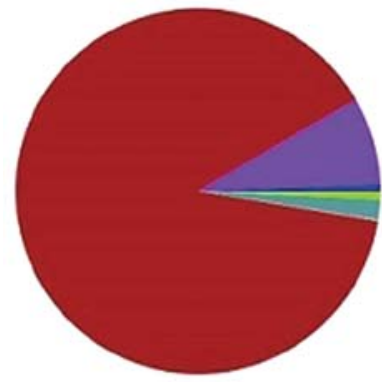

Milk stasis

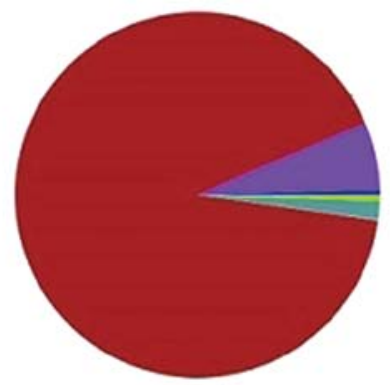

Milk stasis plus tumor
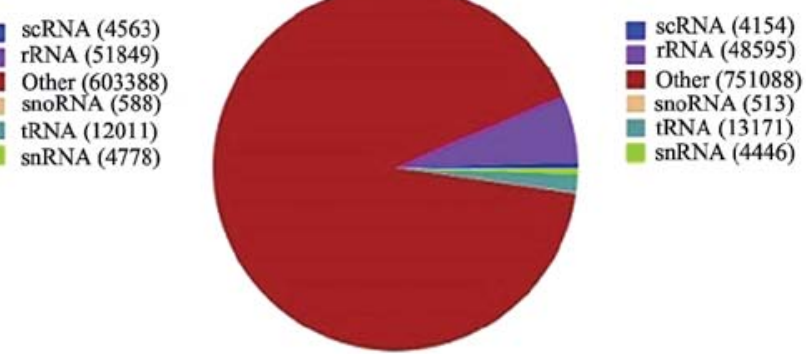

Figure 5. Subtype of microRNA (miRNA) identified in two groups.

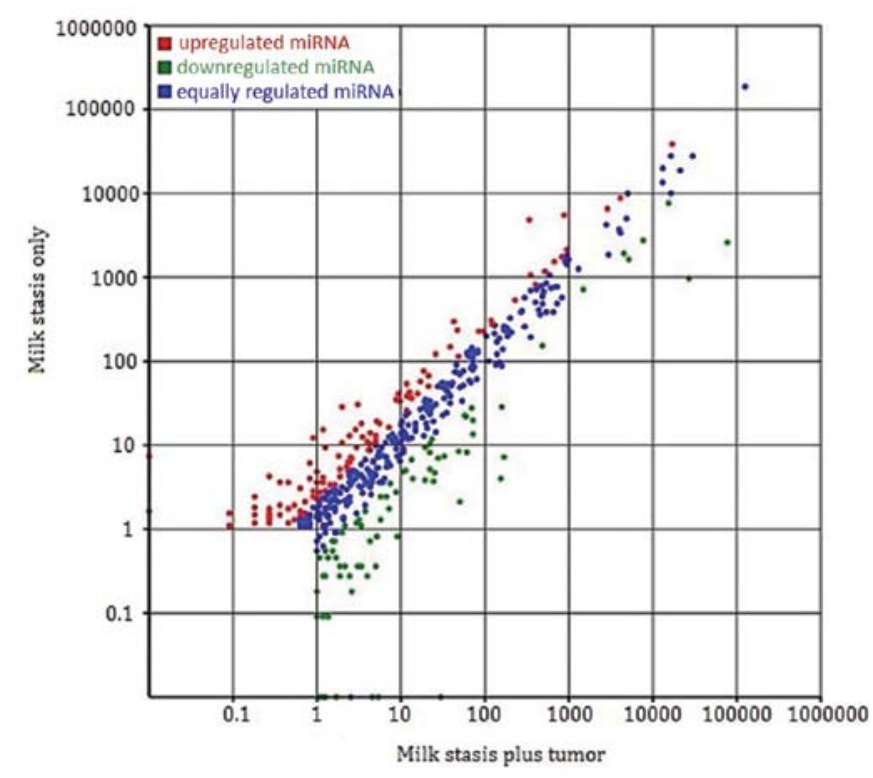

Figure 6. Differential expression. suppressors (hsa-miR-21-5p, hsa-miR-423-5p, hsa-let-7b-5p, hsa-let-7c, hsa-let-7f-5p, hsa-miR-23a-3p, hsa-miR-101-3p, hsa-miR-143-3p, hsa-let-7i-5p), while hsa-miR-140-3p had oncogenic function (Fig. 7C).

Twenty immune-related miRNAs, which are reported in the milk of $<11$ months of lactation were compared. Six of the 20 were significantly downregulated in the milk stasis plus breast neoplasm group, however, mir-92-3p and mir-92-5p which have an oncogenic function were significantly upregulated (Fig. 7D).

The differential miRNA profiling between the two groups suggested that miRNA involved in milk stasis may play important role in breast carcinogenesis.

Verification of the expression of miRNAs in milk stasis. To confirm that miRNAs are abundant in milk stasis, we used quantitative PCR to quantify the expression of three miRNAs (miR-140, miR-21 and let-7a) in randomly selected 20 milk samples, 10 of which were milk stasis samples, and the remaining 10 milk stasis plus tumor samples. Consistent with 

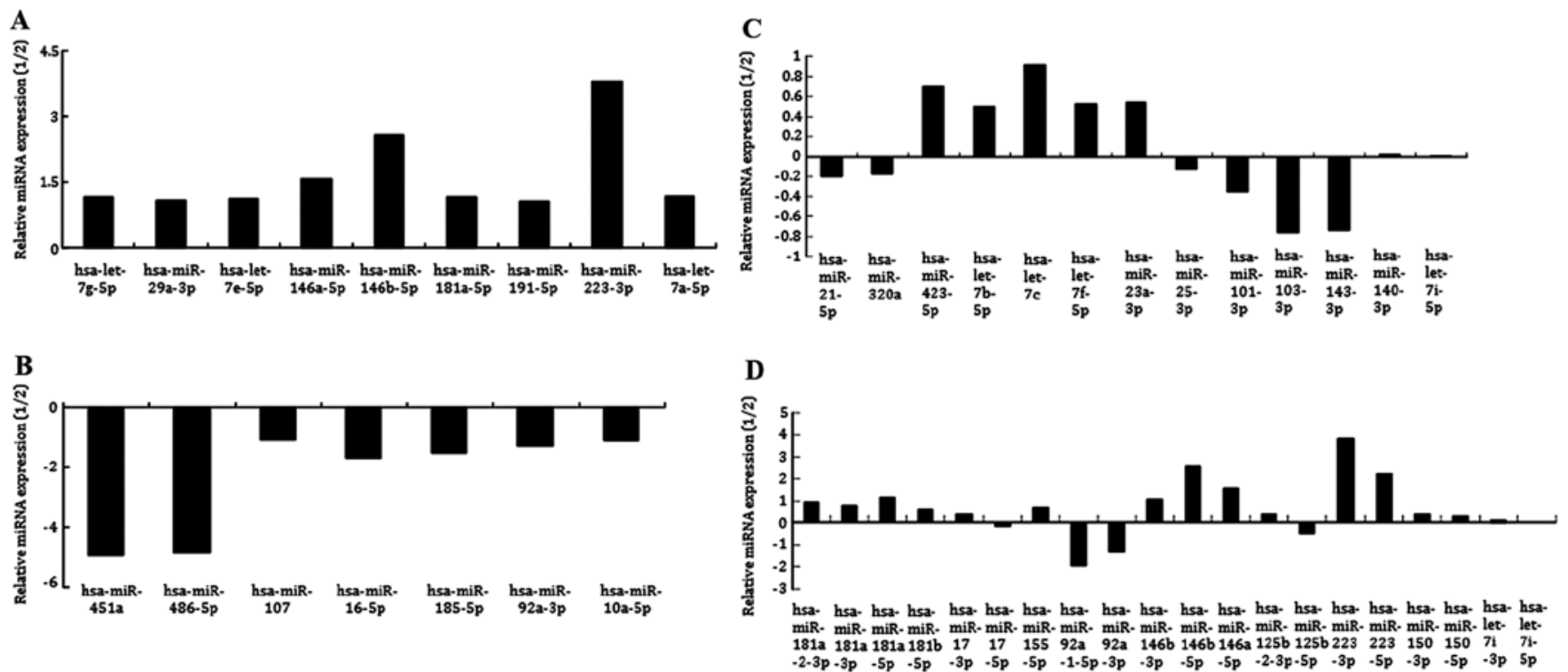

Figure 7. Differentially expressed microRNA (miRNA) reported between the two groups. (A) Downregulated miRNAs in the milk stasis plus tumor group, (B) upregulated miRNAs in the milk stasis plus tumor group, (C) highly expressed miRNAs in the two groups, (D) immune-related miRNAs which are reported in the milk of $<11$ months of lactation in the two groups. Y-axis 1: milk stasis, 2: milk stasis plus tumor.
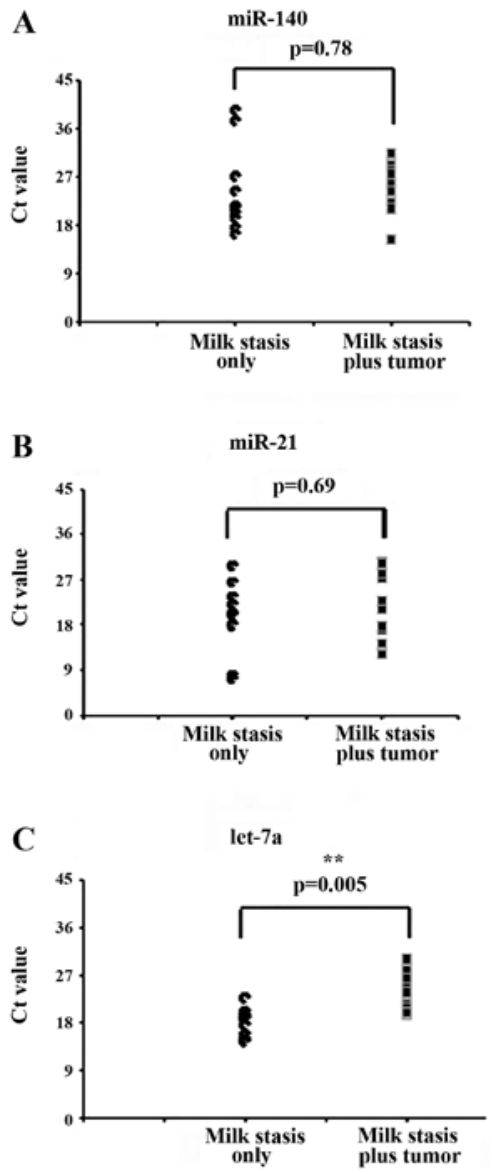

Figure 8. Differentially expressed microRNA (miRNA) confirmed by quantitative PCR.

our sequencing data, miR-140, miR-21 and let-7a were highly expressed in the selected samples (Fig. 8). Thus, these results confirm that miRNAs robustly exist in milk from milk stasis patients.
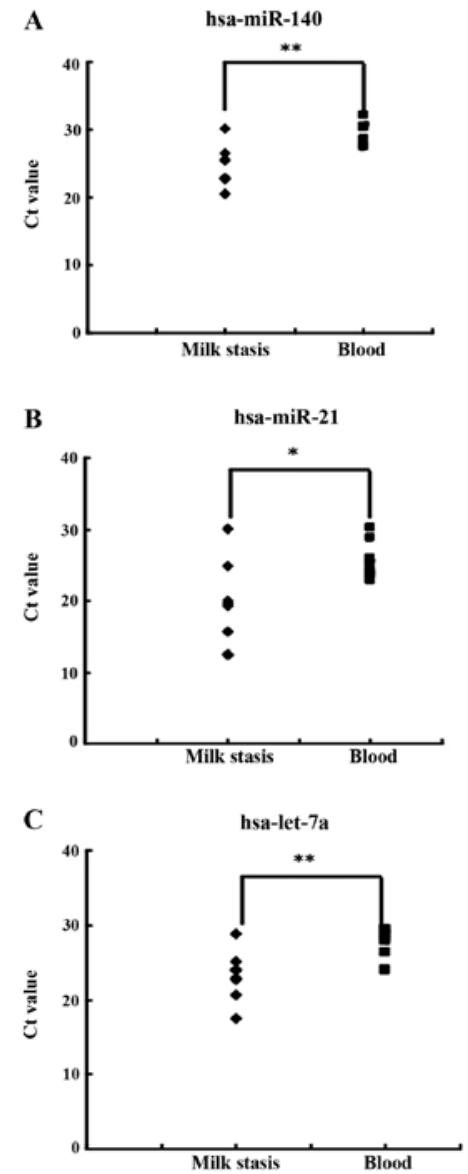

Figure 9. MicroRNA (miRNA) level in blood and milk from milk stasis patients.

miRNAs are more abundant in milk stasis than in the blood. Identification of biomarkers that predict or prevent breast cancer is important for breast cancer patients. Due to the abundance of miRNAs in milk stasis, we examined whether milk 
miRNAs are better biomarkers for breast cancer than blood miRNAs. We selected seven patients and determined miRNAs (miR-140, miR-21 and let-7a) both in the milk and the blood. Results showed that miR-140, miR-21 and let-7a were more abundant in the milk than in the blood (Fig. 9). The results suggest that miRNAs in the milk from milk stasis patients may be more sensitive biomarkers.

\section{Discussion}

Previous studies have focused on gaining a better understanding of breast carcinogenesis; however, the underlying molecular mechanisms remain to be determined $(31,32)$. It is well recognized that the tumor microenvironment plays critical roles in breast cancer development $(33,34)$. Milk acts as an important microenvironment of breast cancer, however, its role in breast carcinogenesis is largely unknown. Milk can remain in the breast over a long period of time after feeding (milk stasis) and the milk remaining in the breast can influence the microenvironment of the breast. However, the exact role of milk stasis in breast carcinogenesis is unknown. To the best of our knowledge, this is the first study on the abundance of miRNAs in milk remaining in breast after feeding. Furthermore, we found that miRNA profiling was different between milk stasis only patients and patients with milk stasis plus breast neoplasm. The different miRNAs profiles suggest that miRNAs in milk remaining in breast may contribute to breast carcinogenesis. We also provided evidence that miRNAs in milk remaining in breast were more easily detected compared to miRNAs in the blood.

Milk consists mainly of water, proteins, lactose, fat, and minerals with wide-ranging chemical, physical and functional activities. Evidence has shown that milk also contains miRNAs (35). Weber et al have reported that miR-509-5p, miR-515-3p, and miR-335 were the most abundant miRNAs in the majority of the body fluid samples including milk, while miR-193b was unique in breast milk (36). Zhou et al reported that miR-148A-3P, miR-30B-5P, let-7f-5p, miR-146B-5P, miR-29A-3P, let-7a-5p, miR-141-3P, miR-182-5P, miR-200A-3P and miR-378-3P were the top 10 miRNAs identified in the milk (26). However, Munch et al showed that miR-148a, let-7a, mir-200c, miR-146b-5p, let-7f, miR-30d, miR-103, let-7b, let-7g and hsa-mir-21 were the most abundant miRNAs present in the milk (24). Consistent with previous studies, we found that lavage from milk stasis patients contained a variety of miRNAs. As the patients included in this study stopped feeding at least three years previously, the miRNAs in the milk were stabilized and affected breast microenviroment over a long period of time, which may cause breast cancer. Profiling therefore is a good biomarker for breast tumors especially the ductal carcinoma in situ.

Kosaka et al systematically analyzed the expression of miRNAs in milk during the first six months and 6-11 months of lactation, and found that the majority of immune-related miRNAs were downregulated during lactation. In this study, we found that the expression of immune-related miRNAs between milk stasis only and milk stasis plus neoplasm groups was different, suggesting that the biological feature of milk stasis is different from lactation. Milk of lactation provides nutrition and immunity to infants and supports their health.
By contrast, milk from milk stasis potentially acts as a risk factor for breast cancer because our profile data showed that many oncogenic miRNAs were highly expressed in the two groups studied and the immune-related miRNAs were almost downregulated.

The let-7 family miRNAs are the most recognized miRNAs in milk from healthy patients (17-19). Consistent with previous reports, we found that let-7 family miRNAs (let-7g-5p, let-7e-5p, let-7a-5p, let-7b-5p, -let-7c, let-7f-5p, let-7i-5p) were abundant in the patients with milk stasis only, although their levels were significantly reduced in patients with milk stasis plus breast tumor. Let-7 family miRNAs are known to play a tumor suppressor function (17-19). Furthermore, a number of other tumor suppressor miRNAs such as miR-29a, miR-146 and miR-223 were downregulated, while oncogenic miRNAs such as miR-451, miR-486, miR-107, miR-92 and miR-10 were upregulated in the milk of milk stasis plus neoplasm patients. These results suggest that the expression switch of oncogenic and tumor suppressor miRNAs in the milk of milk stasis patients contributes to breast carcinogenesis. However, the specific function of individual miRNA identified in milk stasis in breast carcinogenesis remains to be addressed.

Another significant finding of this study is that miRNAs in breast lavage of milk stasis patients are more easily detected than miRNAs in the blood. Thus miRNAs in breast lavage of milk stasis may be better biomarkers to predict the risk of breast cancer.

In summary, although the sample size included in the current study was limited, we provided several novel and noteworthy findings. To the best of our knowledge, this is the first study on miRNA prolifing of breast lavage of milk stasis patients. Our data suggest that miRNAs in milk from milk stasis patients contribute to breast carcinogenesis and they are more sensitive biomarkers of breast cancer than miRNAs in the blood.

\section{References}

1. Ballard $\mathrm{O}$ and Morrow AL: Human milk composition: nutrients and bioactive factors. Pediatr Clin North Am 60: 49-74, 2013

2. Smetherman DH: Screening, imaging, and image-guided biopsy techniques for breast cancer. Surg Clin North Am 93: 309-327, 2013.

3. Langlands FE, Horgan K, Dodwell DD and Smith L: Breast cancer subtypes: response to radiotherapy and potential radiosensitisation. Br J Radiol 86: 20120601, 2013.

4. Benson JR and Jatoi I: The global breast cancer burden. Future Oncol 8: 697-702, 2012.

5. Reeder JG and Vogel VG: Breast cancer prevention. Cancer Treat Res 141: 149-164, 2008.

6. Collaborative Group on Hormonal Factors in Breast Cancer: Breast cancer and breastfeeding: collaborative reanalysis of individual data from 47 epidemiological studies in 30 countries, including 50302 women with breast cancer and 96973 women without the disease. Lancet 360: 187-195, 2002.

7. Russo J and Russo IH: Susceptibility of the mammary gland to carcinogenesis. II. Pregnancy interruption as a risk factor in tumor incidence. Am J Pathol 100: 497-512, 1980.

8. Yang L and Jacobsen KH: A systematic review of the association between breastfeeding and breast cancer. J Womens Health (Larchmt) 17: 1635-1645, 2008.

9. Carmichael AR: Obesity as a risk factor for development and poor prognosis of breast cancer. BJOG 113: 1160-1166, 2006.

10. Warmuth MA, Sutton LM and Winer EP: A review of hereditary breast cancer: from screening to risk factor modification. Am J Med 102: 407-415, 1997.

11. Schwab M, Claas A and Savelyeva L: BRCA2: a genetic risk factor for breast cancer. Cancer Lett 175: 1-8, 2002. 
12. Narod SA: BRCA mutations in the management of breast cancer: the state of the art. Nat Rev Clin Oncol 7: 702-707, 2010.

13. Bartel DP: MicroRNAs: genomics, biogenesis, mechanism, and function. Cell 116: 281-297, 2004.

14. Ambros V: The functions of animal microRNAs. Nature 431 : 350-355, 2004.

15. Castañeda CA, Agullo-Ortuño MT, Fresno Vara JA, Cortes-Funes H, Gomez HL and Ciruelos E: Implication of miRNA in the diagnosis and treatment of breast cancer. Expert Rev Anticancer Ther 11: 1265-1275, 2011.

16. Harquail J, Benzina S and Robichaud GA: MicroRNAs and breast cancer malignancy: an overview of miRNA-regulated cancer processes leading to metastasis. Cancer Biomark 11: 269-280, 2012

17. Hu X, Guo J, Zheng L, et al: The heterochronic microRNA let-7 inhibits cell motility by regulating the genes in the actin cytoskeleton pathway in breast cancer. Mol Cancer Res 11: 240-250, 2013.

18. Qian P, Zuo Z, Wu Z, et al: Pivotal role of reduced let-7g expression in breast cancer invasion and metastasis. Cancer Res 71: 6463-6474, 2011.

19. Yu F, Yao H, Zhu P, et al: let-7 regulates self renewal and tumorigenicity of breast cancer cells. Cell 131: 1109-1123, 2007.

20. Castilla MÁ, Díaz-Martín J, Sarrió D, et al: MicroRNA-200 family modulation in distinct breast cancer phenotypes. PLoS One 7: e47709, 2012.

21. Ng EK, Li R, Shin VY, et al: Circulating microRNAs as specific biomarkers for breast cancer detection. PLoS One 8: e53141, 2013.

22. Kosaka N, Izumi H, Sekine K and Ochiya T: microRNA as a new immune-regulatory agent in breast milk. Silence 1: 7, 2010.

23. Sun Q, Chen X, Yu J, Zen K, Zhang CY and Li L: Immune modulatory function of abundant immune-related microRNAs in microvesicles from bovine colostrum. Protein Cell 4: 197-210, 2013.

24. Munch EM, Harris RA, Mohammad M, et al: Transcriptome profiling of microRNA by Next-Gen deep sequencing reveals known and novel miRNA species in the lipid fraction of human breast milk. PLoS One 8: e50564, 2013.
25. Song XM, Jiang JF and Jiang YQ: Progress on miRNA in mammal breast milk. Yi Chuan 34: 1233-1241,2012 (In Chinese).

26. Zhou Q, Li M, Wang X, et al: Immune-related microRNAs are abundant in breast milk exosomes. Int J Biol Sci 8: 118-123, 2012

27. Izumi H, Kosaka N, Shimizu T, Sekine K, Ochiya T and Takase M: Bovine milk contains microRNA and messenger RNA that are stable under degradative conditions. J Dairy Sci 95: 4831-4841, 2012

28. Abou-Dakn M, Richardt A, Schaefer-Graf U and Wöckel A: Inflammatory breast diseases during lactation: milk stasis, puerperal mastitis, abscesses of the breast, and malignant tumors-current and evidence-based strategies for diagnosis and therapy. Breast Care (Basel) 5: 33-37, 2010.

29. Green KA, Nielsen BS, Castellino FJ, Rømer J and Lund LR: Lack of plasminogen leads to milk stasis and premature mammary gland involution during lactation. Dev Biol 299: 164-175, 2006.

30. Noble MS and Hurley WL: Effects of secretion removal on bovine mammary gland function following an extended milk stasis. J Dairy Sci 82: 1723-1730, 1999.

31. Rakha EA and Chan S: Metastatic triple-negative breast cancer. Clin Oncol (R Coll Radiol) 23: 587-600, 2011.

32. Kumar DH and Kutty MK: Review of stem cell deregulation and breast cancer: an emerging hypothesis. Indian J Pathol Microbiol 55: 147-153, 2012.

33. Ward C, Langdon SP, Mullen P, et al: New strategies for targeting the hypoxic tumour microenvironment in breast cancer. Cancer Treat Rev 39: 171-179, 2013.

34. Artacho-Cordón A, Artacho-Cordón F, Ríos-Arrabal S, Calvente I and Núñez MI: Tumor microenvironment and breast cancer progression: a complex scenario. Cancer Biol Ther 13: 14-24, 2012.

35. Walker A: Breast milk as the gold standard for protective nutrients. J Pediatr 156 (2 Suppl): S3-S7, 2010.

36. Weber JA, Baxter DH, Zhang S, et al: The microRNA spectrum in 12 body fluids. Clin Chem 56: 1733-1741, 2010. 